\title{
Systemic sclerosis in association with multiple primary pulmonary malignancy - a marker of internal malignancy?
}

\author{
M.J.D. Goodfield and L.G. Millard
}

Department of Dermatology, University Hospital, Nottingham, UK.

\begin{abstract}
Summary: Multiple pulmonary malignancy is uncommon and has not been reported in association with systemic sclerosis. We now report such a case in which it is likely that the connective tissue disease occurred as a marker of the internal malignancy. Systemic sclerosis is not generally a reliable indicator of malignant change, but it is possible that in patients who are immunosuppressed, either therapeutically or inherently, the onset of systemic sclerosis should prompt investigation for an underlying cause.
\end{abstract}

\section{Introduction}

Certain connective tissue diseases are regarded as markers of internal malignancy. ${ }^{1}$ This is particularly true of dermatomyositis, ${ }^{2}$ but systemic sclerosis has been similarly discussed. ${ }^{3}$ Opinions are mixed, some authors claiming a definite relationship between systemic sclerosis and internal carcinoma, ${ }^{4,5}$ whilst others regard their co-existence as fortuitous. ${ }^{1}$ Pulmonary malignancy has been linked particularly with systemic sclerosis, ${ }^{5}$ although some doubt even this relationship. ${ }^{6}$

Multiple primary carcinoma are uncommon, but well described. ${ }^{7}$ The association between multiple pulmonary squamous tumours and systemic sclerosis has not so far been reported, although in one large review of the occurrence of malignancy in systemic sclerosis, 3 cases of multiple primary tumours of different organs occurred. ${ }^{8}$ We now report a case of multiple primary squamous tumours of the lung preceding the onset of rapidly progressive systemic sclerosis.

\section{Case report}

A man of 57 presented with a one month history of a productive cough, with no haemoptysis. He had been a heavy smoker until 7 years previously. He worked as a maintenance fitter with the National Coal Board, but had never worked underground. He had no exposure to polyvinyl chloride. His only medication was trinitrin for mild angina.

Correspondence: M.J.D. Goodfield, M.A., M.R.C.P., Department of Dermatology, General Infirmary at Leeds, Great George St., Leeds LS1 3EX, UK.

Accepted: 16 June 1988
Examination revealed an abnormality in the right $\mathrm{O}$ lung field, and chest X-ray showed a large mass in the right upper lobe with cavitation, and two $Z$ further masses inferiorly (Figure 1). Haematological ${ }_{\Phi}$ and biochemical investigation was normal. Bronchoscopy showed three separate tumours in different bronchi with the appearances of primagy tumours, all histologically squamous.

Right pneumonectomy was performed wif success and a good recovery. Histology on the operative specimen confirmed the pre-operatives findings.

Four years later, he presented with a 2-month

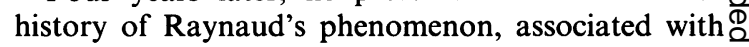
thickening of the skin of the hands. Examination $\overrightarrow{\overrightarrow{0}}$ confirmed typical sclerodactyly with trophic 3 changes of the finger pulps, and dilated nail fold $\bar{F}$ capillaries. Further investigation showed the presence of an homogeneous anti-nuclear antibody (IgG, 1 in 200, IgM, 1 in 100), with negative antiDNA antibodies. Further immunological studies:were normal, as were a barium meal and oeso-3. phageal pressure studies. Chest X-ray was unchanged from post-operatively, and lung function tests were compatible with his surgery, with noo evidence of a restrictive defect.

Six months later, he developed dyspepsia and을 oesophageal reflux, confirmed by barium meal and pressure studies to be due to systemic sclerosis. His 0 vascular symptoms deteriorated, requiring in- $N$ patient treatment, and his general condition worsened with weight loss and general malaise. $A_{\sigma}^{\omega}$ chest X-ray showed a small peripheral lesion in in the left mid-zone, again with the appearance of a $\frac{0}{\Phi}$ primary tumour. There was no clinical suggestion of aspiration, and further investigation excluded ${ }_{0}^{+}$

(C) The Fellowship of Postgraduate Medicine, $1988 \frac{\text { 윰 }}{\mathrm{D}}$ 

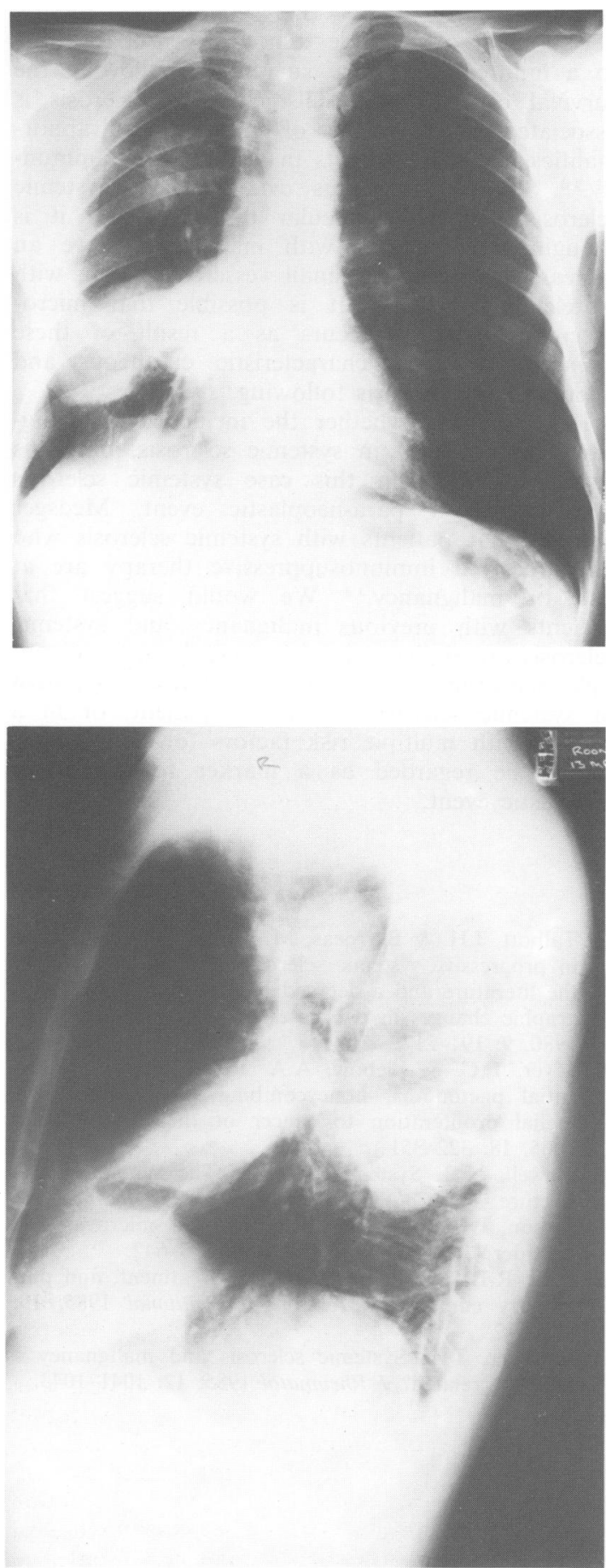

Figure 1 PA and lateral chest $X$-ray at initial presentation showing three mass lesions in the right lung field. tuberculosis, pyogenic infection, and pulmonary embolism. The pulmonary lesion enlarged and cavitated (Figure 2) and the patient died 2 months later at home of fulminating pneumonia. There was no post-mortem.

\section{Discussion}

Multiple tumours occur in about $1 \%$ of all pulmonary malignancies, ${ }^{7}$ and so, although uncommon, the presence of four primaries in this patient is not in itself extraordinary. The aetiology of the tumours is clear since the patient was a heavy smoker, but it is the co-existence with systemic sclerosis that may be of significance.

The incidence of malignancy in systemic sclerosis is of the order of $12 \%$, an incidence similar to that in the general population. ${ }^{3}$ The site of tumours is probably also similar to that in control populations, with an excess of gynaecological and breast malignancy due to the female preponderance in systemic sclerosis, ${ }^{3}$ although other reports stress the temporal relationship between the onset of malignancy

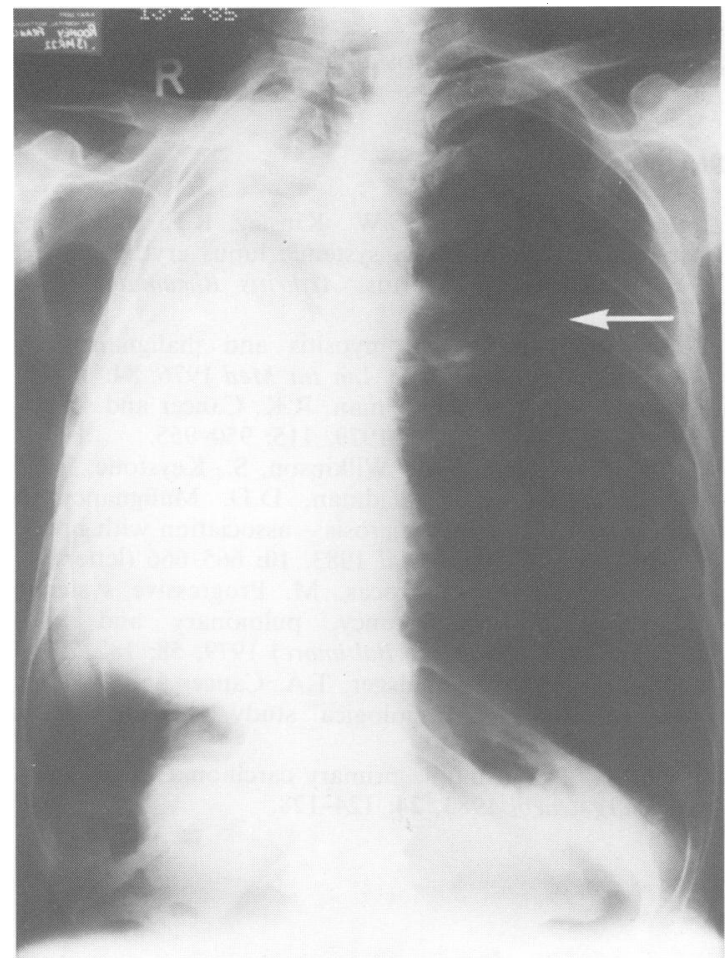

Figure 2 PA chest radiograph showing cavitating lesion in the left lung field (arrowed). 
and of systemic sclerosis. ${ }^{8}$ The frequency of pulmonary malignancy is also disputed, some authors believe it to be the most common site for malignancy in systemic sclerosis, ${ }^{5}$ whilst others feel that this is simply sampling error. ${ }^{3,6}$

Certainly, pulmonary tumours, particularly adeno-carcinomata, occur in any fibrotic lung tissue, whatever the aetiology, ${ }^{9}$ and can thus follow long standing pulmonary involvement in systemic sclerosis. It seems likely that other histological types of pulmonary neoplasia are not found in excess in systemic sclerosis.

The nature of any relationship between systemic sclerosis and malignancy has been much discussed, and variously described as fortuitous, causative and para-neoplastic. In this case, the occurrence of systemic sclerosis in a man, which is unusual, and occurring at a time when he was developing a further tumour, makes it likely that this was at least a para-neoplastic event. It is possible that the fourth lesion was a secondary tumour (though this appears unlikely), but the relationship of connective tissue disease to active malignancy still holds. The patient had no other risk factors for the development of systemic sclerosis, and there was no clinical evidence of abnormal production of humoral agents such as serotonin.

There are several possible explanations for the

\section{References}

1. Lewis, R.B., Castor, C.W., Kinsley, R.E. et al. Frequency of neoplasia in systemic lupus erythematosus and rheumatoid arthritis. Arthritis Rheumatol 1976, 19: 1256-1261.

2. Barnes, B.E. Dermatomyositis and malignancy. A review of the literature. Ann Int Med 1976, 84: 68-76.

3. Duncan, S.C. \& Winkelman, R.K. Cancer and scleroderma. Arch Dermatol 1979, 115: 950-955.

4. Lee, P., Alderdice, C., Wilkinson, S., Keystone, E.C., Urowitz, M.B. \& Gladman, D.D. Malignancy in progressive systemic sclerosis - association with breast carcinoma. J Rheumatol 1983, 10: 665-666 (letter).

5. Talbott, J.H. \& Barrocas, M. Progressive systemic sclerosis and malignancy, pulmonary and nonpulmonary. Medicine (Baltimore) 1979, 58: 182-207.

6. Roumm, A.D. \& Medsger, T.A. Cancer and systemic sclerosis. An epidemiological study. Arthritis Rheum 1985, 28: 1336-1340.

7. Chung, T.S. Multiple primary carcinoma of the lung. J Surg Oncol 1983, 24: 124-128. concurrence of these two conditions. Firstly, 3 development of multiple tumours is probably due $\stackrel{\varnothing}{\varrho}$ to a failure of immune surveillance allowing the $\measuredangle$ survival of neoplastic cells. Systemic sclerosis is $\underset{\vec{\rho}}{\vec{\rho}}$ associated with a number of immunological abnormalities, including defects in cell-mediated immunity. ${ }^{10}$ Secondly, there is evidence that systemic $\frac{\bar{\sigma}}{\bar{D}}$ sclerosis is a microvascular disease, ${ }^{11}$ and it is $\vec{\odot}$ thought that patients with malignancy have an increased tendency to small vessel occlusion, with $\%$ platelet activation. ${ }^{12}$ It is possible that micro- $\overrightarrow{0}$ vascular occlusion occurs as a result of these changes, with the characteristic cutaneous and $\vec{\omega}$ internal organ fibrosis following.

It is doubtful whether the incidence of malig- -00 nancy is increased in systemic sclerosis, but it is also likely that in this case systemic sclerosis $\$$ occurred as a para-neoplastic event. Medsger or believes that patients with systemic sclerosis who. have received immunosuppressive therapy are at $\infty$ risk of malignancy. ${ }^{13}$ We would suggest that $\odot$ patients with previous malignancy and systemic sclerosis are similarly at risk, and those with mul- $\vec{z}$ tiple malignancy particularly so. The development $Z$ of systemic sclerosis in such a patient, or in a patient with multiple risk factors for malignancy, should be regarded as a marker for a further $\mathbb{\Phi}$ neoplastic event.

8. Talbott, J.H. \& Barrocas, M. Carcinoma of the lung in progressive systemic sclerosis: a tabular review of the literature and a detailed report of the roentgenographic changes in two cases. Semin Arthritis Rheum 1980, 9: 191-217.

9. Myer, E.C. \& Liebow, A.A. Relationship of interstitial pneumonia, honeycombing and atypical epithelial proliferation to cancer of the lung. Cancer 1965, 18: $322-351$.

10. Rowell, N.R. Systemic sclerosis. The Watson Smith Lecture. J R Coll Physicians London 1984, 19: 23-28.

11. Jayson, M.V. Systemic sclerosis - a microvascular disorder? J R Soc Med 1983, 76: 635-642.

12. Hull, R.D. Venous thrombosis - treatment, and pulmonary embolism. Medicine International 1985, 19:

13. Medsger, T.A. Systemic sclerosis and malignancy -
(3) are they related? J Rheumatol 1985, 12: 1041-1043. 